\title{
A Plakophilin-1 Gene Mutation in an Egyptian Family with Ectodermal Dysplasia-Skin Fragility Syndrome
}

\author{
Ebtesam M. Abdalla ${ }^{a}$ Cristina Has ${ }^{b}$ \\ a Department of Human Genetics, Medical Research Institute, Alexandria University, Alexandria, Egypt; \\ ${ }^{b}$ Department of Dermatology, University Medical Center Freiburg, Freiburg, Germany
}

\section{Key Words}

Ectodermal dysplasia-skin fragility syndrome - Egypt .

Plakophilin-1 gene

\begin{abstract}
Ectodermal dysplasia-skin fragility syndrome (ED-SFS) is a rare genodermatosis caused by mutations in the PKP1 gene, encoding the desmosomal plaque protein plakophilin-1. Since its initial description in 1997, few individuals with this disorder have been reported to date. Here, we present the first Egyptian cases of ED-SFS, carrying a novel homozygous mutation in the PKP1 gene. Direct sequencing of the amplified DNA from the affected cases disclosed a G-to-T transversion at nucleotide position c.203-1 within intron 1 of PKP1 (c.203-1G>T). To the best of our knowledge, this mutation has not been previously described in the databases.

(c) 2014 S. Karger AG, Basel
\end{abstract}

Ectodermal dysplasia-skin fragility syndrome (EDSFS) is a rare genodermatosis caused by mutations in the PKP1 gene, encoding the desmosomal plaque protein plakophilin-1 [McGrath et al., 1997, 1999]. This autosomal recessive disorder represents the first described inherited disorder of desmosomes. Loss-of-function mutations in PKP1 lead to widespread skin fragility, which is the prominent feature of the disease. Nearly all cases also have palmoplantar keratoderma with painful fissures that are often disabling. The disease also manifests with nail dystrophy and abnormal hair growth in the form of hypotrichosis or complete alopecia [Whittock et al., 2000; Hamada et al., 2002]. Occasionally, depending on the particular PKP1 mutations, the hair may be woolly rather than reduced. Other variable clinical features include reduced sweating, scattered scale-crusts on the trunk and limbs, pruritus, and recurrent systemic infections [McGrath and Mellerio, 2010]. Since its initial description in 1997, few individuals with this disorder have been reported to date. According to the Orphanet Report Series 2012 (http://www.orpha.net/orphacom/cahiers/docs/GB/ Prevalence_of_rare_diseases_by_decreasing_prevalence_or_cases.pdf), 10 published cases and families with this syndrome are registered, and the Human Gene $\mathrm{Mu}-$ tation Database (HGMD Professional 2013.3, http:// www.hgmd.cf.ac.uk/ac/index.php) contains 13 PKP1 mutations. Here, we report the first Egyptian cases of EDSFS, carrying a novel homozygous mutation in the PKP1 gene.

\section{KARGER}

E-Mail karger@karger.com

www.karger.com/msy (c) 2014 S. Karger AG, Base

$1661-8769 / 14 / 0056-0304 \$ 39.50 / 0$
Ebtesam M. Abdalla

Department of Human Genetics, Medical Research Institute

Alexandria University, 165 El-Horreya Avenue El-Hadra

21561 Alexandria (Egypt)

E-Mail drebtesamabdalla@yahoo.com 


\section{Clinical Report}

The 5- and 4-year-old female probands are the offspring of healthy first-cousin Egyptian parents and are the only affected members of the entire family (fig. 1A). Both patients had an average physical growth and normal intellectual developmental milestones, although they showed some motor delay because of the skin condition. The disease proceeded similarly in the 2 sibs; they were both born at full term with normal skin and scalp hair. Three days after birth, the condition started with extensive blistering all over the body, more severe on the limbs, while milder on the face and trunk. After rupture of the blisters, the underlying skin healed without scarring. At that time, the condition was thought to be a form of epidermolysis bullosa. Few months later, nail dystrophy started with recurrent falling off of the nails. Concurrently, the normal original hair was lost and replaced by scanty abnormal hair. At the age of $\sim 1$ year, the skin blistering improved dramatically, although the skin was still fragile and scalded very easily on minimal trauma. When the sibs were 2 years old, hyperkeratosis of the palms and soles became evident. Dentition started at the age of 1 year; the teeth were initially normal and then became dysplastic after 2 years. Moreover, the patients suffered from frequent chest infections that necessitated hospitalization. On examination, the patients displayed sparse friable scalp hair, curly eyelashes, cheilitis with perioral fissuring, dysplastic teeth, yellowish thickened nails with subungual hyperkeratosis, and palmoplantar hyperkeratosis, which was very severe in the soles, with painful fissuring. We also noted numerous erosions and crusts over the face and limbs (fig. 1B, C).

Since the constellation of clinical findings displayed by both patients was typical of ED-SFS, we obtained peripheral blood samples from the affected girls and their parents and performed DNA extraction. All coding exons and flanking intronic regions of the PKP1 gene (GenBank NM_001005337.2) were amplified by PCR. Direct sequencing of the amplified DNA from the affected cases disclosed a G-to-T transversion at nucleotide position c.203-1 within intron 1 of PKP1 (c.203-1G>T). The patients were shown to carry the mutation in a homozygous state, while the parents were heterozygous carriers. To the best of our knowledge, this variant has not been described before in the Human Gene Mutation Database, and it is not referenced in the Single Nucleotide Database (dbSNP 141, http://www.ncbi.nlm.nih.gov/projects/SNP/), in 1000 Genomes (http://www.1000genomes.org/) or on the Exome Variant Server (http://evs.gs.washington.edu/EVS).

\section{Discussion}

In 1997, McGrath et al. described the first case of a human genetic disease resulting from inherited mutations in a structural component of desmosomes. The clinical features included skin erosions and peeling with histological evidence of widening of intercellular spaces between keratinocytes, extending from the suprabasal to the granular layer [McGrath et al., 1997]. However, there were also early changes in hair and nails, suggesting that $P K P 1$ had an additional role in the development of certain
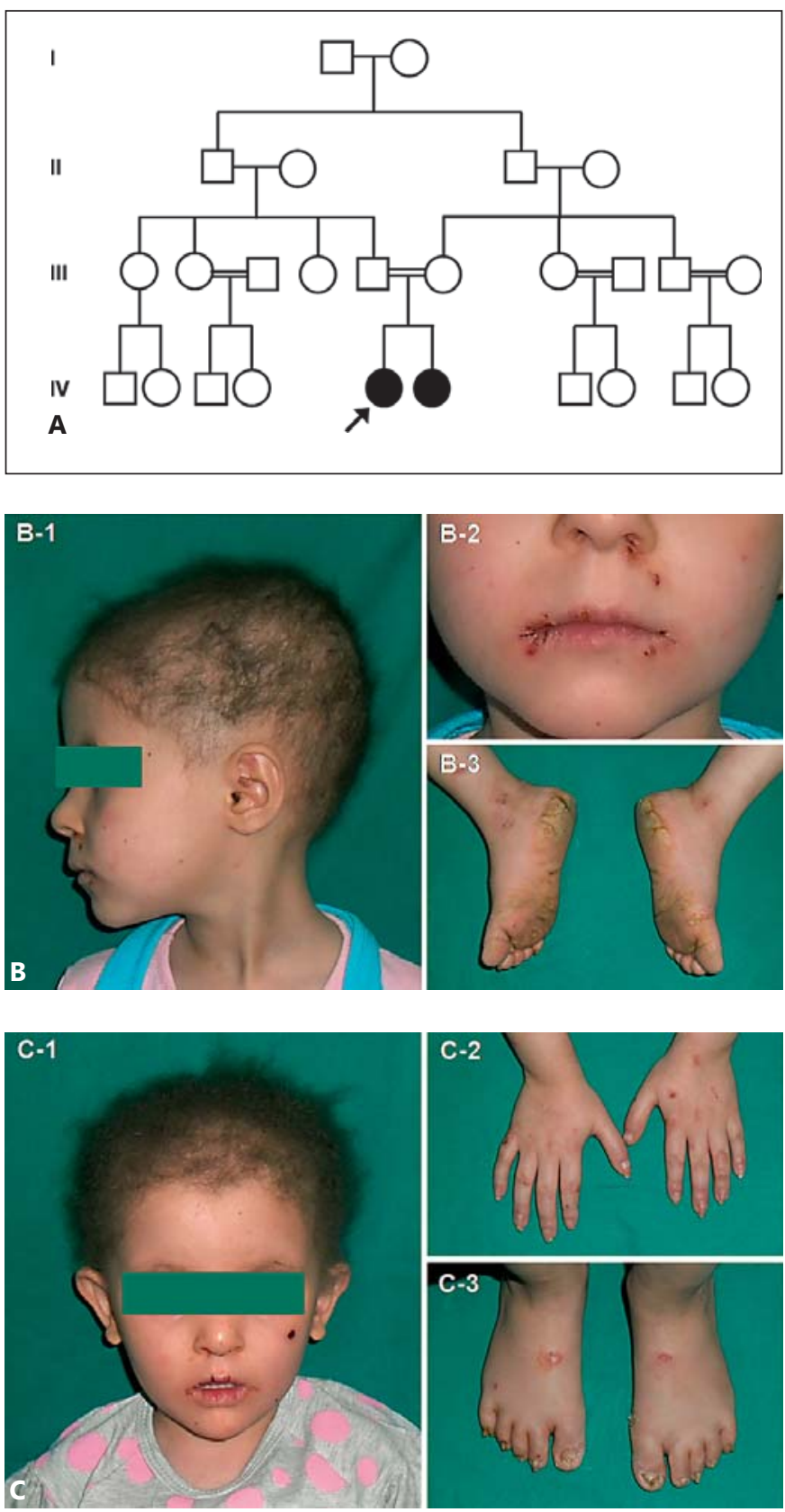

Fig. 1. Pedigree $(\mathbf{A})$ and clinical presentation $(\mathbf{B}, \mathbf{C})$ of the 2 patients with ED-SFS. A The index patient is indicated by an arrow. B Clinical features of the 5-year-old index patient (IV-5). B-1 Sparse, short, thin, and lusterless scalp hair and numerous skin erosions. B-2 Facial erosions, which are most prominent around the mouth with perioral fissuring. B-3 Extensive plantar hyperkeratosis with fissures. C Clinical features of the younger patient (IV-6). C-1 Sparse, lusterless and woolly scalp hair, skin erosion over the left cheek, and cheilitis with perioral fissuring. C-2 Dysplastic fingernails and multiple skin erosions. C-3 Toenail plate thickening and yellowish discoloration as well as superficial erosions over the foot dorsum. 
ectodermal structures. Although all cases of ED-SFS reported so far showed similar clinical abnormalities, subtle differences between their phenotypic manifestations were noted [Hamada et al., 2002; Steijlen et al., 2004]. Hypohidrosis and cheilitis were constant features, while the extent of the alopecia and the degree of disability associated with the skin fissures varied between patients. This clinical diversity probably reflects the impact of the individual PKP1 mutations on the levels of functional plakophilin-1 protein. However, the paucity of cases with this syndrome makes it difficult to establish accurate genotype-phenotype correlations.

The mutation c.203-1G>A was detected in a case of ED-SFS, and it was shown to generate 2 aberrant transcripts, predicted to lead to premature termination codons and absence of plakophilin-1 expression [McGrath et al., 1999]. Subsequently, the same mutation was recurrently reported as disease-causing in other patients from different origins [Sprecher et al., 2004]. In this report, the patients, who belonged to a consanguineous family of Arab Christian origin, were found to carry a G>T transversion at the same nucleotide position. Thus, the nucleotide position c.203-1 is likely to represent a mutational hot spot. Interestingly, splice site mutations are overrepresented in this gene. The various outcomes of leaky splice site anomalies, allowing residual protein expression, may correlate with improvement of the clinical phenotype [Wessagovit and McGrath, 2005]. For that reason, we intend to follow the progress of our patients with this rare genodermatosis. Given the clinical similarities between the present cases and the previously described male and female patients from different races [Zheng et al., 2005; Ersoy-Evans et al., 2006; Adhe et al., 2011; Boyce et al., 2012], it seems that neither sex nor ethnic origin significantly influence the phenotypic expression of mutations in the PKP1 gene.

In summary, ED-SFS is a rare inherited skin disorder. We describe the first Egyptian patients suffering from ED-SFS, caused by a splice site mutation within the PKP1 gene. This result provides further evidence for the previously established role of PKP1 mutations in the pathophysiology of ED-SFS and expands the database of $P K P 1$ mutations.

\section{Acknowledgements}

This study was supported by the German Research Foundation grant HA 5663/2-1 to C.H. We are grateful to the patients and their parents for their cooperation.

\section{References}

Adhe VS, Dongre AM, Khopkar US: Ectodermal dysplasia-skin fragility syndrome. Indian J Dermatol Venereol Leprol 77:503-506 (2011).

Boyce AE, McGrath JA, Techanukul T, Murrell DF, Chow CW, et al: Ectodermal dysplasiaskin fragility syndrome due to a new homozygous internal deletion mutation in the PKP1 gene. Australas J Dermatol 53:61-65 (2012).

Ersoy-Evans S, Erkin G, Fassihi H, Chan I, Paller AS, et al: Ectodermal dysplasia-skin fragility syndrome resulting from a new homozygous mutation, $888 \mathrm{delC}$, in the desmosomal protein plakophilin 1. J Am Acad Dermatol 55: 157-161 (2006).

- Hamada T, South AP, Mitsuhashi Y, Kinebucji T, Bleck $\mathrm{O}$, et al: Genotype-phenotype correlation in skin fragility-ectodermal dysplasia syndrome resulting from mutations in plakophilin 1. Exp Dermatol 11:107-114 (2002).
McGrath JA, Mellerio JE: Ectodermal dysplasiaskin fragility syndrome. Dermatol Clin 28: 125-129 (2010).

-McGrath JA, McMillan JR, Shemanko CS, Runswick SK, Leigh IM, et al: Mutations in the plakophilin 1 gene result in ectodermal dysplasia/skin fragility syndrome. Nat Genet 17 : 240-244 (1997).

McGrath JA, Hoeger PH, Christiano AM, McMillan JR, Mellerio JE, et al: Skin fragility and hypohidrotic ectodermal dysplasia resulting from ablation of plakophilin 1. Br J Dermatol 140:297-307 (1999).

- Sprecher E, Molho-Pessach V, Ingber A, Sagi E, Indelman M, Bergman R: Homozygous splice site mutations in PKP1 result in loss of epidermal plakophilin 1 expression and underlie ectodermal dysplasia/skin fragility syndrome in two consanguineous families. J Invest Dermatol 122:647-651 (2004).
Steijlen PM, van Steensel MA, Jansen BJ, Blokx W, van de Kerkhof PC, et al: Cryptic splicing at a non-consensus splice-donor in a patient with a novel mutation in the plakophilin 1 gene. J Invest Dermatol 122:1321-1324 (2004).

Wessagovit V, McGrath JA: Clinical and molecular significance of splice site mutations in the plakophilin 1 gene in patients with ectodermal dysplasia-skin fragility syndrome. Acta Derm Venereol 85:386-388 (2005).

-Whittock NV, Haftek M, Angoulvant N, Wolf F, Perrot H, et al: Genomic amplification of the human plakophilin 1 gene and detection of a new mutation in ectodermal dysplasia/skin fragility syndrome. J Invest Dermatol 115: 368-374 (2000).

Zheng R, Bu DF, Zhu XJ: Compound heterozygosity for new splice site mutations in the plakophilin 1 gene (PKP1) in a Chinese case of ectodermal dysplasia-skin fragility syndrome. Acta Derm Venereol 85:394-399 (2005). 